\title{
Intracellular pH dynamics regulates intestinal stem cell fate
}

Yi Liu ${ }^{1,2}$, Efren Reyes ${ }^{3} \dagger$, David Castillo-Azofeifa ${ }^{3} \dagger$, Ophir D. Klein ${ }^{3}$, Todd Nystul ${ }^{2 *} \dagger$, Diane L. Barber ${ }^{1 * \dagger}$

\section{Affiliations:}

${ }^{1}$ Department of Cell and Tissue Biology, University of California San Francisco; San Francisco, CA 94143, USA.

${ }^{2}$ Departments of Anatomy, University of California San Francisco; San Francisco, CA 94143, USA.

${ }^{3}$ Program in Craniofacial Biology and Department of Orofacial Sciences, University of California San Francisco; San Francisco, CA 94143, USA.

*Co-corresponding authors. Email: diane.barber@ucsf.edu; todd.nystul@ucsf.edu $\dagger$ These authors contributed equally to this work

Summary: Emerging evidence is revealing critical roles of intracellular $\mathrm{pH}(\mathrm{pHi})$ in development (1-4), but it remains unclear whether $\mathrm{pHi}$ regulates stem cell fate specification. We find that $\mathrm{pHi}$ dynamics is a key regulator of cell fate in the mouse intestinal stem cell lineage. We identify a pHi gradient along the intestinal crypt axis and find that dissipating this gradient inhibits crypt budding due to loss Paneth cell differentiation. Mechanistically, decreasing pHi 
biases intestinal stem cell fate toward the absorptive and away from the secretory lineage, by regulating the activity of the lineage transcription factor Atoh1. Our findings reveal a previously unrecognized role for $\mathrm{pHi}$ dynamics in the specification of cell fate within an adult stem cell lineage.

\section{Introduction:}

Intracellular $\mathrm{pH}(\mathrm{pHi})$ dynamics is emerging as a key regulator of diverse cell behaviors, including epithelial to mesenchymal transition (5), transformation and dysplasia (6,7), and differentiation of embryonic and adult stem cells (2-4). A role for $\mathrm{pHi}$ dynamics in the differentiation and lineage specification of mammalian adult stem cells, however, has not been reported. We investigated this possibility in the mouse intestinal stem cell (ISC) lineage using organoid cultures derived from the small intestine. Intestinal organoids are a self-sustaining in vitro epithelial model that recapitulates many features of in vivo mammalian intestinal epithelium, including self-renewing stem cells at the base of budding crypts, a distinct crypt/villus architecture, and differentiation and lineage specification of stem cell progeny ( 8 11).

\section{Results:}

\section{A pHi gradient in small intestinal organoids}

To quantify the pHi of cells in the ISC lineage, we generated organoids stably expressing a genetically encoded pHi biosensor, mCherry-SEpHluorin $(6,13,14)$. We acquired ratiometric (SEpHluorin/mCherry) images at days 1 and 3 of organoid growth (Fig. 1, A and B, and fig. S1A) and calibrated fluorescent ratios to pHi by perfusing with nigericin-containing buffers of known $\mathrm{pH}$ values at the end of each imaging set (14). We found that at day 1 of organoid growth 
all cells have a similar pHi but at day 3 a pHi gradient developed along the crypt (Fig. 1, B and C). We quantified pHi values of $\sim 7.2$ in the ISCs and Paneth cells, $\sim 7.4$ in the progenitor cells on the crypt column and $\sim 7.5$ on the crypt neck region (Fig. 1C). Ratiometric time-lapse imaging also revealed a similar pHi gradient, with higher $\mathrm{pHi}$ in cells within the column and neck than in

the crypt base (Fig. 1D, and movie S1). In summary, we find that ISCs in the crypt base have a lower pHi than more differentiated cells along the crypt column and in the crypt neck region. (Fig. 1D, and movie S1). These differences in pHi (Fig. 1C) raised the question of whether increased pHi is associated with ISC differentiation and fate decision. membrane Na-H exchanger NHE1, a ubiquitously expressed acid extruder and a key regulator of pHi dynamics in mammalian tissues, including the intestine (12). Additionally, the Drosophila ortholog $d N h e 2$ is necessary for the pHi gradient within the ovarian follicle stem cell lineage (2). Consistent with this prediction, we found that organoids treated with 5-(N-Ethyl-N-isopropyl)amiloride (EIPA), a selective pharmacological inhibitor of NHE1 (15) but not other NHE isoforms, had a significantly lower $\mathrm{pHi}$ in ISCs and adjacent non-Paneth cells compared with controls at day 3 (Fig. 1, B and C). In contrast, pHi remained unchanged in ISCs between controls and EIPA-treated organoids at day 1. Hence, inhibiting NHE1 activity reduced pHi in different cell types and disrupted the developed pHi gradient.

\section{NHE1 activity is necessary for crypt growth}

To determine whether a pHi gradient is functionally significant, we tested the role of NHE1regulated pHi on organoid development. In control organoids, crypts began budding by day 1 
after passage, with more and elongated buds by day 3 (Fig. 2A, and movie S2A). In contrast, inhibiting of NHE1 activity with EIPA or by doxycycline (DOX) inducible CRISPR-Cas9 gene silencing (Fig. 2C) significantly reduced the number of crypts that budded (Fig. 2, B and D). When crypt buds did form in organoids treated with EIPA, they were routinely not retained (Fig. 2A, and movie S2B). Loss of budding was restored after removing EIPA (Fig. 2, E and F), indicating that organoids remain viable with EIPA (fig. S1B). To test whether loss of budding is due to the absence of crypt cells, we immunolabeled for the hyaluronic acid receptor CD44, a crypt marker expressed throughout the crypt cell population, including Lgr5 $5^{+}$ISCs, Paneth cells, and crypt progenitors $(16-18)$. We found that $\mathrm{CD}_{4} 4^{+}$cell clusters were retained in organoids that lack NHE1 activity (Fig. 2G). We also identified Paneth cells, as indicated by lysozyme (LYZ) immunolabeling, within the $\mathrm{CD} 44^{+}$population of cells, suggesting that these $\mathrm{CD} 44^{+}$clusters are unbudded crypts. Taken together, these observations indicate that loss of NHE1 activity disrupts crypt budding but does not eliminate crypt cell populations.

To test whether inhibiting NHE1 activity affected cell proliferation, we incubated control and EIPA-treated organoids with 5-ethynyl-2'-deoxyuridine (EdU) and assayed for EdU incorporation. We found no significant difference in the rate of EdU incorporation by CD44 cells in EIPA-treated organoids compared with controls (Fig. 2, H and I). However, Lgr5 expression was significantly reduced in EIPA-treated organoids at day 3 (Fig. 2K), suggesting that inhibition of NHE1 reduces the ISC number. Consistent with this possibility, we observed by live-cell imaging that $\operatorname{Lgr} 5-D T R-G F P$ expression decreases over a 3-day period in $\operatorname{Lgr} 5^{\text {DTR- }}$ GFP organoids treated with EIPA (Fig. 2A, and movie S2B). To quantify this effect, we used $\operatorname{Lgr} 5^{\text {DTR-GFP }}$ organoids to identify ISCs and immumolabeled day 3 organoids for CD44 to identify all crypt cells. Indeed, we found a significant decrease in the percentage of CD44 ${ }^{+}$cells 
that are $\operatorname{Lgr} 5^{+}$with EIPA compared with controls. Lgr $5^{+}$ISCs will differentiate by default in the absence of Wnt-signaling $(19,20)$, and loss of $\operatorname{Lr} 5^{+}$ISCs causes crypt loss in organoids (21) and in vivo (22-25). Therefore, these data suggest that inhibiting NHE1 activity attenuates crypt budding and growth by interfering with the maintenance of $\operatorname{Lgr} 5^{+}$ISCs.

\section{Exogenous WNT rescues crypt budding impaired with loss of NHE1 activity}

ISC self-renewal requires the Wnt signaling pathway agonist, R-spondin1 (Rspo1), and Wnt3a, a canonical pathway ligand $(8,19,20,26,27)$. We hypothesized that the reduced number of $\operatorname{Lg} 5^{+}$ ISCs in organoids that lack NHE1 activity may be due to decreased Wnt pathway activity. To test this hypothesis, we first immunolabeled organoids for EphB2, a target of Wnt pathway activity that is expressed mostly in crypt base cells $(28,29)$. EphB2 immunolabeling was predominantly toward the bottom of crypts in control organoids and was markedly reduced in EIPA-treated organoids (Fig. 3E, and fig. S3). We next assayed for the effects of exogenous Rspo1 or Wnt3A on control or EIPA-treated organoids. We found that adding exogenous Wnt3a or increased amounts of Rspo1 restored budded crypts in EIPA-treated and NHE1-silenced organoids to numbers quantified in controls (Fig. 3, A-D). Likewise, expression of EphB2 was also restored by adding Wnt3a and Rspo1 in organoids lacking NHE1 activity (Fig. 3E, and fig. S3). $(8,30)$. Hence, we asked whether reduced Wnt pathway activity in organoids with loss of NHE1 activity is due to a loss of Paneth cell function. To address this question, we used a single cell based in vitro reconstitution assay that allows directly testing niche functions of Paneth cells $(8$, 
30-32). Reconstituting the ISC niche by mixing single WT $\mathrm{Lgr}^{+}$ISCs with single NHE1silenced Paneth cells in the absence of Wnt3a did not reduce the efficiency of organoid formation compared with the Wnt3a-treated WT $\mathrm{Lgr}^{+}$ISCs alone or the WT $\mathrm{Lgr}^{+}$ISC-WT Paneth cell pair (Fig. 3F). This result indicates that loss of NHE1 activity does not impair Wnt secretion by Paneth cells. We next asked whether loss of NHE1 activity changes the number of Paneth cells in the crypt cell population (CD44 ${ }^{+}$cluster). In control organoids, the number of Paneth cells significantly increased from day 1 to day 3 (Fig. 3H). In contrast, with EIPA Paneth cell number from day 1 to 3 remained unchanged (Fig. $3 \mathrm{H}$ ). Collectively, these data indicate that loss of NHE1 activity reduces Wnt-dependent Lgr $5^{+}$ISC maintenance at least in part by decreasing the number of Paneth cells in crypts.

\section{Inhibiting NHE1 activity impairs Lgr5 ${ }^{+}$ISC lineage specification toward the secretory but not absorptive cell fate}

We next determined whether disrupting the NHE1-generated pHi gradient impairs specification of ISC lineages. The ISC lineage bifurcates into the absorptive lineage, which primarily consists of enterocytes, and the secretory lineage, which includes Paneth cells, enteroendocrine cells, and goblet cells $(10,11)$. Using qPCR for cell type specific markers we found that expression of the absorptive cell marker Alpil was unchanged in EIPA-treated cultures, whereas expression of all three secretory cell markers ( $L y z$ for Paneth cells, $C h g A$ for enteroendocrine cells, and $M u c 2$ for goblet cells) was significantly decreased (Fig. 4A). To further test effects on secretory cell fate, we used $A$ toh $1^{\text {CreERT2 }}$;Rosa $26^{\text {tdTomato }}$ organoids (33) to perform lineage tracing on Atoh $1^{+}$ secretory cells. We tracked tdTomato ${ }^{+}$(secretory lineage label) cells during organoid development (Fig. 4E, and movies S3, A and B). We observed newly differentiated Atoh $1^{+}$ 
secretory cells in control organoids (Fig. 4E, and movie S3A) but not in organoids treated with EIPA (Fig. 4E, and movie S3B), as indicated by the number of tdTomato ${ }^{+}$cells detected on each day (Fig. 4F) and by the net increase in the number of Atoh $1^{+}$cells within the same crypt region (Fig. 4G). In addition, we quantified the number of newly generated Paneth cells within the tdTomato $^{+}$lineages from day 1 to day 2 by scoring for cells containing dense granules, which are visible with bright-field microscopy (Fig. 4B). In control organoids, the number of crypt Paneth cells significantly increased from day 1 to day 2, as expected, whereas with EIPA the number of tdTomato ${ }^{+}$Paneth cells remained constant (Fig. 4, C and D).

We next tested whether loss of NHE1 activity impairs the differentiation of $\mathrm{Lgr}^{+}$ISCs into secretory progenitors, which is the first cell fate decision during the lineage specification. We used Lgr5 ${ }^{+}$ISC lineage tracing with $\operatorname{Lgr} 5^{\mathrm{CreER}}$;Rosa $26^{\mathrm{RFP}}$ organoids (33), which allows tracing Lgr $^{+}$ISC progeny in the crypt as well as in the villus (fig. S4A), including secretory progenitors (Fig. 4H), Paneth cells (fig. S4C), and enterocytes (Fig. 4K). In control organoids, RFP ${ }^{+}$ISC daughter cells in the $\mathrm{CD} 44^{+}$region differentiate to secretory progenitors marked by the enrichment of Delta-like 1 ligand (D111) (34) (Fig. 4H). In contrast, in EIPA-treated organoids the frequency (Fig. 4I) and number (Fig. 4J) of $\mathrm{RFP}^{+}, \mathrm{Dll}^{+}$cells in the $\mathrm{CD}^{4} 4^{+}$region were significantly reduced. Consistent with the reduced number of Paneth cell (Fig. 3H, and Fig. 4A) and their production (Fig. 4, C and D), loss of NHE1 activity significantly decreased the number of $\mathrm{LYZ}^{+}$Paneth cells in the $\mathrm{RFP}^{+}$clones generated from $\mathrm{Lgr}^{+}$ISCs (fig. S4, C-E). Even in EIPA-treated organoids in which nearly all the cells were $\mathrm{RFP}^{+}$, Paneth cells were still negative for RFP (fig. S3B), further confirming a defect in the fate decision of the $\operatorname{Lr} 5^{+}$ISC daughter cells toward Paneth cells. These results indicate that loss of NHE1 activity and a pHi gradient impair lineage specification into the secretory cell fate. Furthermore, we show in control 
organoids that the new Paneth cells accumulate in the growing crypt, consistent with the idea that replenishment is important for maintaining a sufficient number of Paneth cells in the crypt (35).

To test whether EIPA treatment impaired specification of the absorptive cell lineage, we used Lgr $5^{\text {CreER}} ;$ Rosa $26^{\mathrm{RFP}}$ lineage tracing to quantify differentiation of $\mathrm{Lgr}^{+}$ISCs into enterocytes, which are a post-mitotic cell in the absorptive lineage marked by the metabolic enzyme Aldolase B (Fig. 4K) (36,37). We found a similar number of $\mathrm{RFP}^{+}$enterocytes in control and EIPA-treated organoids at days 2 and 3 (Fig. 4, L and M), indicating that loss of NHE1 activity does not impair differentiation to enterocytes. These data are consistent with our observation that control and EIPA-treated organoids have a similar transcript abundance of Alpil, which is an absorptive lineage marker (Fig. 4A), and suggest a robust transient amplification of absorptive progenitors, which is consistent with our proliferation data (Fig. 2I). Because the $\operatorname{Lgr} 5^{+}$ISC pool decreases with loss of NHE1 activity (Fig. 2, J and K), a similar number of RFP ${ }^{+}$(lineage label), Aldolase $\mathrm{B}^{+}$cells seen in the control and EIPA-treated organoids (Fig. 4M) suggests the possibility that differentiation into enterocytes is enhanced with EIPA. Collectively, our data reveal that NHE1 activity and NHE1-regulated pHi dynamics control the cell-fate decision of $\mathrm{Lgr}^{+}$ISCs toward the secretory but not the absorptive lineage.

To determine how the loss of NHE1 activity impairs specification of the secretory lineage, we assayed for the expression of Notch pathway targets. In the ISC lineage, lateral inhibition causes daughter cells just downstream from the ISCs to adopt a state of either high or low Notch pathway activity. High levels of Notch signaling promote the expression of Hes 1 , which represses the secretory lineage transcription factor, Atoh1, and thus allows for differentiation 
toward the absorptive cell fate (12,38-40). Conversely, in cells with low Notch pathway activity, low levels of HES1 permits Atoh1 expression, which promotes the secretory cell fate. Atohl forms a positive feedback loop by reinforcing its own expression and promoting the expression of Dll1 and Dll4 (41), thus enabling lateral inhibition of the secretory fate in neighboring cells. We observed a small but statistically significant decrease in Hes 1 expression in EIPA-treated organoids compared with controls (Fig. 4N), suggesting that inhibiting NHE1 activity may initially bias daughter cells toward the secretory cell fate. However, we did not see a concomitant increase in Atohl expression (Fig. 4N), indicating that this next step in specification of the secretory cell fate is blocked. Consistent with this, we found that Atohl ${ }^{\text {CreERT2 }}$ labeled a similar number of cells in the control and EIPA-treated conditions at the first day of lineage tracing (Fig. 4F), but these labeled lineages did not upregulate D111 (Fig. 4, H-J) and did not differentiate into Paneth cells (Fig. 4, A-D, and fig S3, B-E) in the EIPA condition. Taken together, these data suggest that loss of NHE1 activity impairs the ability of Atoh1 to induce the secretory cell fates.

\section{Discussion:}

Our findings indicate a previously unreported role of NHE1 activity and pHi dynamics in stem cell lineage specification in mammalian small intestinal organoids (Fig. 4O). Combined with our previous reports that increased pHi enables mouse embryonic and Drosophila adult stem cell models $(2,3)$, and a recent report that increased pHi promotes melanocyte differentiation (4), our current results add mouse intestinal organoids as an additional model to highlight an increasingly evident role of $\mathrm{pHi}$ dynamics in stem cell biology. New to this paradigm, however, is our finding that blocking increased pHi also impairs a selective lineage in the intestinal epithelium, with an impaired specification of the secretory but not absorptive cell fate. Our findings also suggest an inverse relationship between $\mathrm{pHi}$ and Wnt pathway gradients (42-44), which is also seen in 
mouse embryonic naïve and primed cells and could be mediated in part by the pHi-regulated stability of b-catenin, which decreases at higher pHi (45). Importantly, a number of diseases that impair intestinal epithelial homeostasis, including cystic fibrosis, cancer, and inflammatory bowel disorders, also have dysregulated pHi dynamics as well as often changes in NHE1 activity or expression $(3,12,46)$. Collectively, our findings open new directions for increased understanding of how stem cell fates are regulated, identifying $\mathrm{pH}$ sensing proteins and pathways controlling lineage specification, and approaches that can be applied to regenerative medicine.

\section{References}

1. M. Oginuma et al., Intracellular $\mathrm{pH}$ controls WNT downstream of glycolysis in amniote embryos. Nature 584, 98-101 (2020).

2. B. Ulmschneider et al., Increased intracellular $\mathrm{pH}$ is necessary for adult epithelial and embryonic stem cell differentiation. J. Cell Biol. 215, 345-355 (2016).

3. K. A. White, B. K. Grillo-Hill, D. L. Barber, Cancer cell behaviors mediated by dysregulated pH dynamics at a glance. J. Cell Sci. 130, 663-669 (2017).

4. M. Benitez, S. Tatapudy, Y. Liu, D. L. Barber, T. G. Nystul, Drosophila anion exchanger 2 is required for proper ovary development and oogenesis. Dev. Biol. 452, 127-133 (2019).

5. D. A. Raja et al., pH-controlled histone acetylation amplifies melanocyte differentiation downstream of MITF. EMBO Rep. 21, (2020).

6. S. R. Amith, J. M. Wilkinson, L. Fliegel, Na+/H+ exchanger NHE1 regulation modulates metastatic potential and epithelial-mesenchymal transition of triplenegative breast cancer cells. Oncotarget 7, 21091-21113 (2016). 
7. T. Sato et al., Single Lgr5 stem cells build crypt-villus structures in vitro without a mesenchymal niche. Nature 459, 262-265 (2009).

8. H. Gehart, H. Clevers, Tales from the crypt: new insights into intestinal stem cells. Nat. Rev. Gastroenterol. Hepatol. 16, 19-34 (2019).

9. J. Sprangers, I. C. Zaalberg, M. M. Maurice, Organoid-based modeling of intestinal development, regeneration, and repair. Cell Death Differ. 28, 95-107 (2021).

10. K. E. Boonekamp, T. L. Dayton, H. Clevers, Intestinal organoids as tools for enriching and studying specific and rare cell types: advances and future directions. J. Mol. Cell Biol. 12, $562-568(2020)$.

11. J. Beumer, H. Clevers, Cell fate specification and differentiation in the adult mammalian intestine. Nat. Rev. Mol. Cell Biol. 22, 39-53 (2021).

12. L. Cao, Z. Yuan, M. Liu, C. Stock, (Patho-)Physiology of $\mathrm{Na}+/ \mathrm{H}+$ Exchangers (NHEs) in the Digestive System. Front. Physiol. 10, (2020).

13. B. K. Grillo-Hill, C. Choi, M. Jimenez-Vidal, D. L. Barber, Increased H+ efflux is sufficient to induce dysplasia and necessary for viability with oncogene expression. Elife 2015, 1-31 (2015).

14. B. K. Grillo-Hill, B. A. Webb, D. L. Barber, Ratiometric Imaging of pH Probes. in Methods in Cell Biology vol. 123 429-448 (Elsevier, 2014).

15. S. F. Pedersen, S. A. King, E. B. Nygaard, R. R. Rigor, P. M. Cala, NHE1 inhibition by amiloride- and benzoylguanidine-type compounds: Inhibitor binding loci deduced from chimeras of NHE1 homologues with endogenous differences in inhibitor sensitivity. J. Biol. Chem. 282, 19716-19727 (2007).

16. J. Zeilstra et al., Deletion of the WNT target and cancer stem cell marker CD44 in Apc(Min/+) mice attenuates intestinal tumorigenesis. Cancer Res. 68, 3655-3661 (2008). 
17. J. Zeilstra et al. Stem cell CD44v isoforms promote intestinal cancer formation in Apc(min) mice downstream of Wnt signaling. Oncogene 33, 665-670 (2014).

18. K. D. Sumigray, M. Terwilliger, T. Lechler, Morphogenesis and Compartmentalization of the Intestinal Crypt. Dev. Cell 45, 183-197.e5 (2018).

19. K. S. Yan et al., The intestinal stem cell markers Bmil and Lgr5 identify two functionally distinct populations. Proc. Natl. Acad. Sci. U. S. A. 109, 466-471 (2012).

20. K. S. Yan et al., Non-equivalence of Wnt and R-spondin ligands during Lgr5 + intestinal stem-cell self-renewal. Nature 545, 238-242 (2017).

21. S. H. Tan et al., A constant pool of Lgr5+ intestinal stem cells is required for intestinal homeostasis. Cell Rep. 34, (2021).

22. F. Kuhnert et al., Essential requirement for Wnt signaling in proliferation of adult small intestine and colon revealed by adenoviral expression of Dickkopf-1. Proceedings of the National Academy of Sciences of the United States of America vol. 101 (Proc Natl Acad Sci U S A, 2004).

23. D. Pinto, A. Gregorieff, H. Begthel, H. Clevers, Canonical Wnt signals are essential for homeostasis of the intestinal epithelium. Genes Dev. 17, 1709-1713 (2003).

24. T. Fevr, S. Robine, D. Louvard, J. Huelsken, Wnt/ $\beta$-Catenin Is Essential for Intestinal Homeostasis and Maintenance of Intestinal Stem Cells. Mol. Cell. Biol. 27, 7551-7559 (2007).

25. J. H. van Es et al., A Critical Role for the Wnt Effector Tcf4 in Adult Intestinal Homeostatic Self-Renewal. Mol. Cell. Biol. 32, 1918-1927 (2012).

26. W. De Lau et al., Lgr5 homologues associate with Wnt receptors and mediate R-spondin signalling. Nature 476, 293-297 (2011). 
27. K. A. Kim et al., Medicine: Mitogenic influence of human R-spondin1 on the intestinal epithelium. Science (80-. ). 309, 1256-1259 (2005).

28. E. Batlle et al., $\beta$-catenin and TCF mediate cell positioning in the intestinal epithelium by controlling the expression of EphB/EphrinB. Cell 111, 251-263 (2002).

29. J. Holmberg et al., EphB Receptors Coordinate Migration and Proliferation in the Intestinal Stem Cell Niche. Cell 125, 1151-1163 (2006).

30. T. Sato et al., Paneth cells constitute the niche for Lgr5 stem cells in intestinal crypts. Nature 469, 415-418 (2011).

31. Ö. H. Yilmaz et al., MTORC1 in the Paneth cell niche couples intestinal stem-cell function to calorie intake. Nature 486, 490-495 (2012).

32. M. J. Rodríguez-Colman et al., Interplay between metabolic identities in the intestinal crypt supports stem cell function. Nature 543, 424-427 (2017).

33. D. Castillo-Azofeifa et al., Atoh1 + secretory progenitors possess renewal capacity independent of Lgr5 + cells during colonic regeneration. EMBO J. 38, (2019).

34. J. H. Van Es et al., Dll1 + secretory progenitor cells revert to stem cells upon crypt damage. Nat. Cell Biol. 14, 1099-1104 (2012).

35. S. R. Amith, J. M. Wilkinson, L. Fliegel, Na+/H+ exchanger NHE1 regulation modulates metastatic potential and epithelial-mesenchymal transition of triplenegative breast cancer cells. Oncotarget 7, 21091-21113 (2016).

36. D. Serra et al., Self-organization and symmetry breaking in intestinal organoid development. Nature 569, 66-72 (2019).

37. I. Lukonin et al., Phenotypic landscape of intestinal organoid regeneration. Nature 586, 275$280(2020)$. 
38. B. Z. Stanger, R. Datar, L. C. Murtaugh, D. A. Melton, Direct regulation of intestinal fate by Notch. Proc. Natl. Acad. Sci. U. S. A. 102, 12443-12448 (2005).

39. S. Fre et al., Notch signals control the fate of immature progenitor cells in the intestine. Nature 435, 964-968 (2005).

40. J. H. Van Es et al., Notch $/ \gamma$-secretase inhibition turns proliferative cells in intestinal crypts and adenomas into goblet cells. Nature 435, 959-963 (2005).

41. Y. H. Lo et al., Transcriptional Regulation by ATOH1 and its Target SPDEF in the Intestine. Cmgh 3, 51-71 (2017).

42. M. Van de Wetering et al., The $\beta$-catenin/TCF-4 complex imposes a crypt progenitor phenotype on colorectal cancer cells. Cell 111, 241-250 (2002).

43. C. Kosinski et al., Gene expression patterns of human colon tops and basal crypts and BMP antagonists as intestinal stem cell niche factors. Proc. Natl. Acad. Sci. U. S. A. 104, 15418$15423(2007)$

44. H. F. Farin et al., Visualization of a short-range Wnt gradient in the intestinal stem-cell niche. Nature 530, 340-343 (2016).

45. K. A. White et al., $\beta$-Catenin is a $\mathrm{pH}$ sensor with decreased stability at higher intracellular pH. J. Cell Biol. 217, 3965-3976 (2018).

46. N. M. Walker et al., Cellular chloride and bicarbonate retention alters intracellular $\mathrm{pH}$ regulation in Cftr KO crypt epithelium. Am. J. Physiol. Gastrointest. Liver Physiol. 310, G70-80 (2016).

47. H. Tian et al., A reserve stem cell population in small intestine renders Lgr5-positive cells dispensable. Nature 478, 255-259 (2011).

48. B. K. Koo et al., Controlled gene expression in primary Lgr5 organoid cultures. Nat. Methods 9, 81-83 (2012). 
49. M. M. Mahe et al., Establishment of Gastrointestinal Epithelial Organoids. Curr. Protoc. Mouse Biol. 3, 217-240 (2013).

50. N. Sachs, Y. Tsukamoto, P. Kujala, P. J. Peters, H. Clevers, Intestinal epithelial organoids fuse to form self-organizing tubes in floating collagen gels. Dev. 144, 1107-1112 (2017).

51. Z. Kabiri et al., Stroma provides an intestinal stem cell niche in the absence of epithelial Wnts. Dev. 141, 2206-2215 (2014).

52. K. L. VanDussen, L. C. Samuelson, Mouse atonal homolog 1 directs intestinal progenitors to secretory cell rather than absorptive cell fate. Dev. Biol. 346, 215-223 (2010).

Acknowledgments: We thank members of the Barber lab and the Nystul lab for useful discussions; members of the Wittmann lab for consultation on imaging; the UCSF Parnassus Flow Core (RRID:SCR_018206) for assistance on flow cytometry; T. Wald and K. Mckinley for organoids culture.

\section{Funding:}

National Institutes of Health grant CA197855 (DLB)

National Science Foundation grant P0538109 (TN, DLB)

National Science Foundation grant 1933240 (TN, DLB, YL)

UCSF Research Allocation Program (TN and DLB)

UCSF RAP Pilot for Established Investigators in Basic and Clinical/Translational Sciences (TN, DLB, YL)

\section{Author contributions:}

Conceptualization: DLB, TN, YL 
Experiment design and data analysis: YL

Investigation: YL, DLB, TN

Funding acquisition: TN, DLB

Supervision: DLB, TN

Writing - original draft: YL

Writing - review \& editing: DLB, TN, ODK, DC, ER

\section{Competing interests: None}

Data and materials availability: All data are available in the main text or the supplementary materials."

\section{Supplementary Materials}

Materials and Methods

Figs. S1 to S4

References (47-52)

Movies S1 to S3 
A

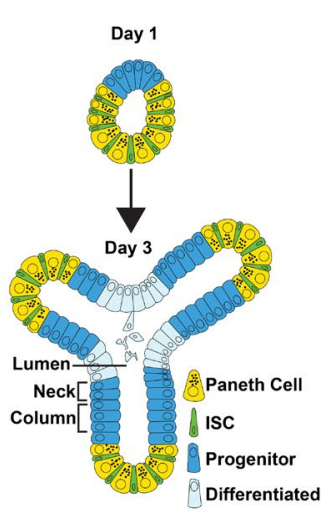

B

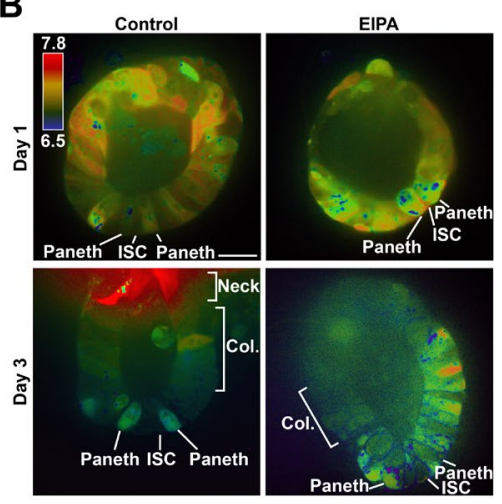

C

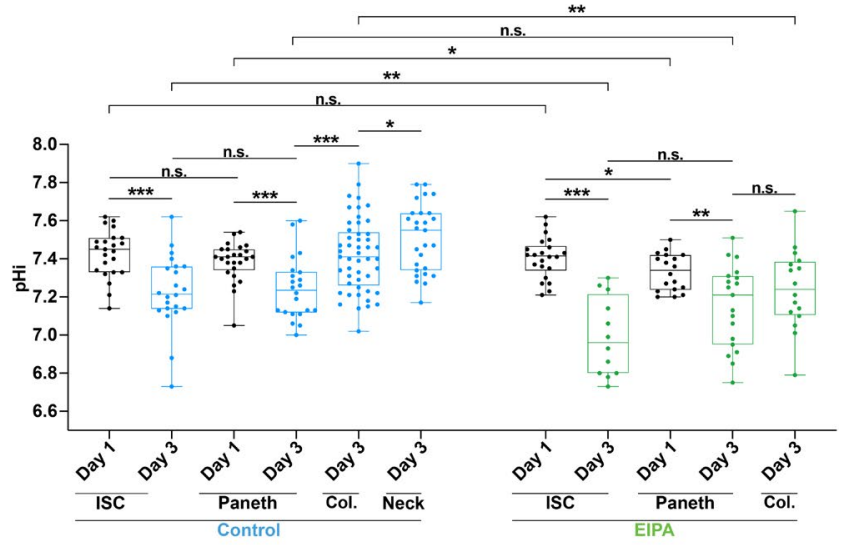

D

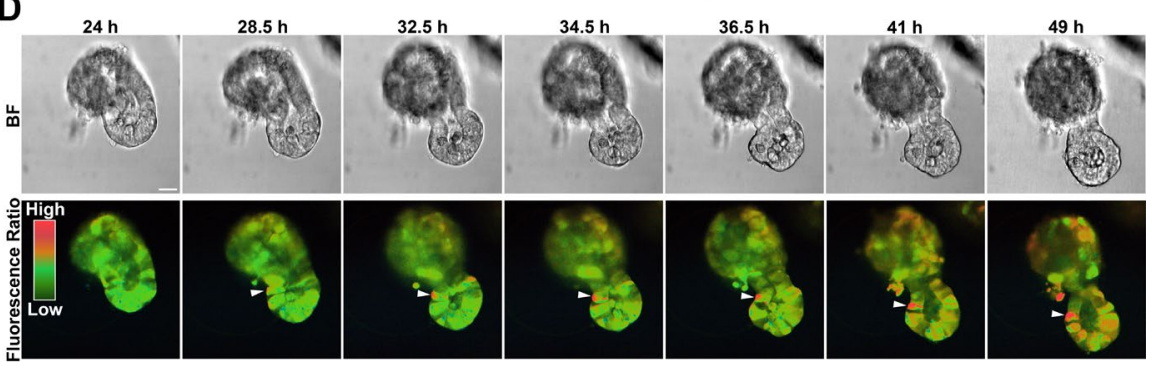

Fig. 1. A pHi gradient develops in small intestinal organoid crypts. (A) Schematic

representation of small intestinal organoids growth. (B to D) pHi in the intestinal crypt. (B) Representative ratiometric images of mCherry-SEpHluorin ratio (SEpHluorin/mCherry) in a control organoid and an organoid treated with $5 \mathrm{mM}$ EIPA from day 1 to day 3 . Images show organoids from a representative 3 preparations. Confocal images of individual fluorescent channels with single-cell resolution (see fig. S1). (C) Values of pHi of different crypt cell types determined by calibrating fluorescent ratios ( $n=3$; Mann-Whitney test). A pHi gradient in the crypts of day 3 control (blue) is attenuated with EIPA (green). Box plots are minimum to 
maximum, the box shows 25 th-75th percentiles, and the central line is the median. (D) Representative frames ( 7 of 51) from 15-hr time-lapse videos of crypt budding started $24 \mathrm{hr}$ after seeding. A cell with a high pHi (ratio) appears in the crypt neck and retained in the crypt column region during crypt growth (arrowhead). BF, bright-field view. Fluorescence Ratio, ratiometric view of mCherry-SEpHluorin. Time-lapse videos of pHi dynamics in crypt budding in movie S1. ${ }^{*} P<0.05, * * P<0.01, * * * P<0.001$. N.s., not statistically significant. All scale bars represent $20 \mathrm{~mm}$. 

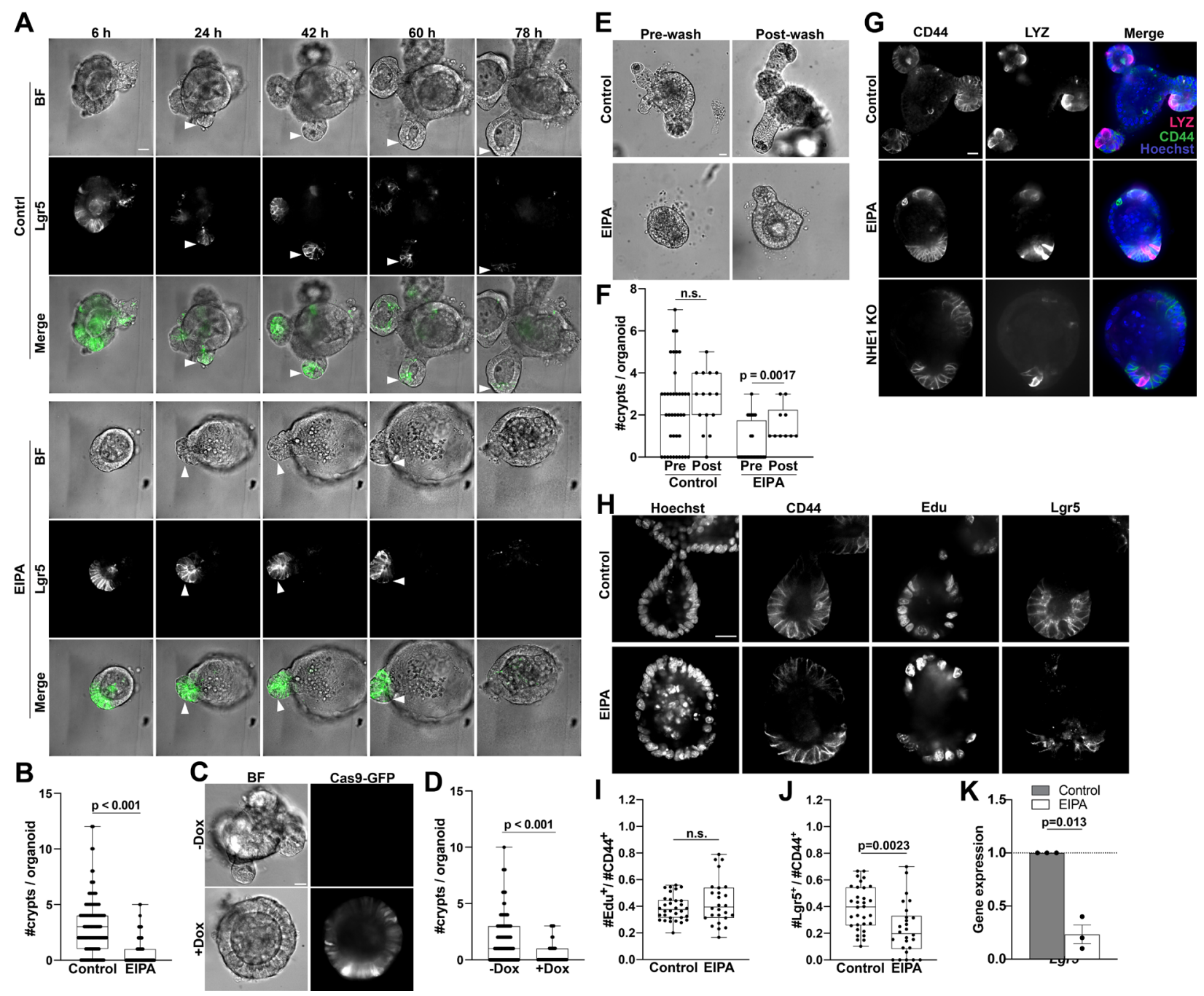

Fig. 2. Loss of NHE1 activity attenuates intestinal crypt budding in organoids. (A)

Representative frames (5 of 25) from 75-hr time-lapse microscopy (see movies S2, A and B) showing crypt budding (arrowhead) in $\operatorname{Lgr} 5^{\text {DTR-GFP }}$ organoids from day 0 (seeding) to day 3 in the absence (Control) and presence of $5 \mathrm{mM}$ EIPA ( $\mathrm{n}=3$ preparations). Control GFP signal is reduced due to photobleaching. BF, bright-field view. Lgr5, Lgr5 ${ }^{+}$ISC. (B) Quantification of crypt number of day 3 organoids treated with or without 5mM EIPA ( $n=4$; Mann-Whitney test). (C and D) Crypt budding in NHE1-silenced organoids. (C) Representative images of inducible CRISPR-Cas9 mediated NHE1-silenced organoids from n=7. (D) Quantification of crypt number in control and NHE1-silenced organoids (n=7; Mann-Whitney test). ( $\mathbf{E}$ and $\mathbf{F}$ ) Reversibility of 
EIPA treatment. (E) Representative images of crypt budding in organoids before and after washing. Pre-wash, 1 day in growth medium (with and without EIPA) after seeding. Post-wash, 1 day after washing and reseeding. (F) Quantification of crypt number in control and EIPAtreated organoids before and after wash (n=3, Mann-Whitney test for each condition; n.s., not statistically significant). (G) Representative images of control organoids and organoids lacking NHE1 activity immunolabeled for CD44 as an index of polarity and lysozyme (LYZ) for Paneth cells ( $\mathrm{n}=3)$. ( $\mathbf{H}$ to $\mathbf{K})$ Crypt proliferation and crypt cell pool with loss of NHE1 activity. (H) Representative images of proliferating $\left(\mathrm{Edu}^{+}\right)$and $\mathrm{LGR}^{+} \mathrm{ISC}$ populations in the crypt region of day 3 organoids $(n=5)$. Quantification of number of proliferating cells $\left(\mathrm{Edu}^{+}\right)(\mathrm{I})$ and $\mathrm{Lgr}^{+}$ISCs (J) in the crypt region $\left(\mathrm{CD} 44^{+}\right)(\mathrm{n}=5$; two-sided student's t-test; n.s., not statistically significant). (K) Relative Lgr5 expression (mean \pm SEM) in control and EIPA-treated organoids $(\mathrm{n}=3$; Wilcoxon test). All box plots are minimum to maximum, the box shows 25 th- 75 th percentiles, and the median is indicated as a central line. Data in all box plots with a statistically significant difference are specified with a $\mathrm{p}$ value. N.s., not statistically significant. All scale bars represent $20 \mathrm{~mm}$. 

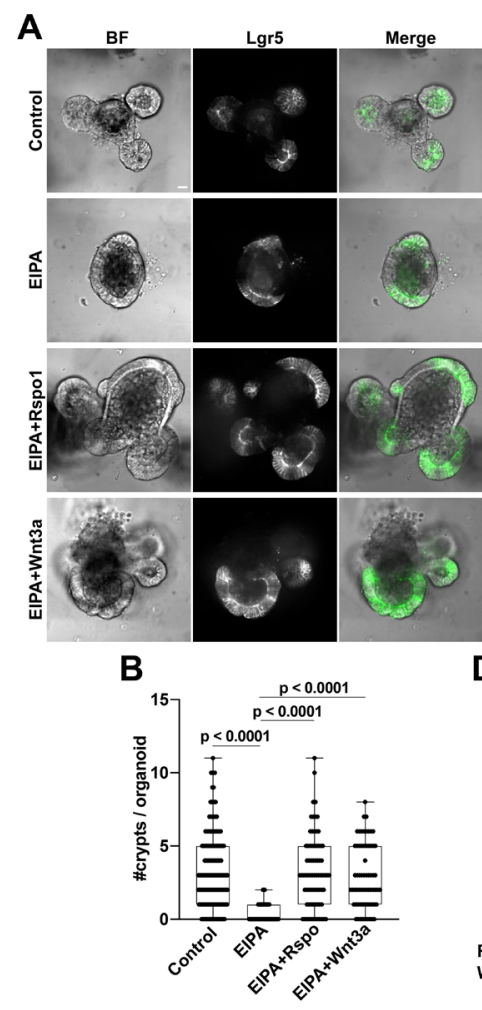
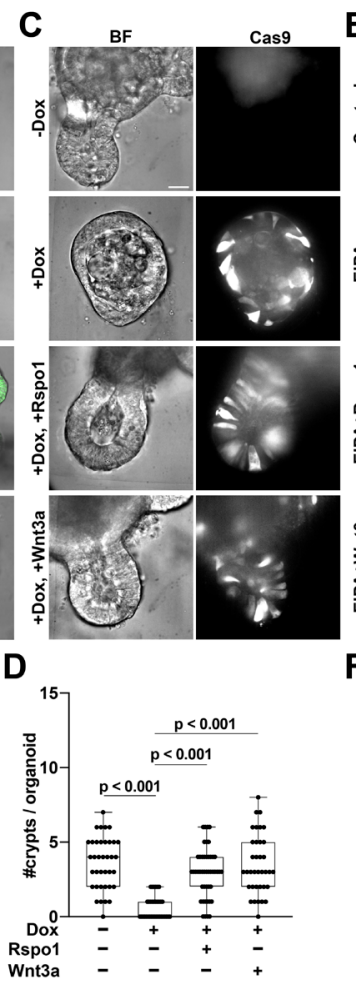
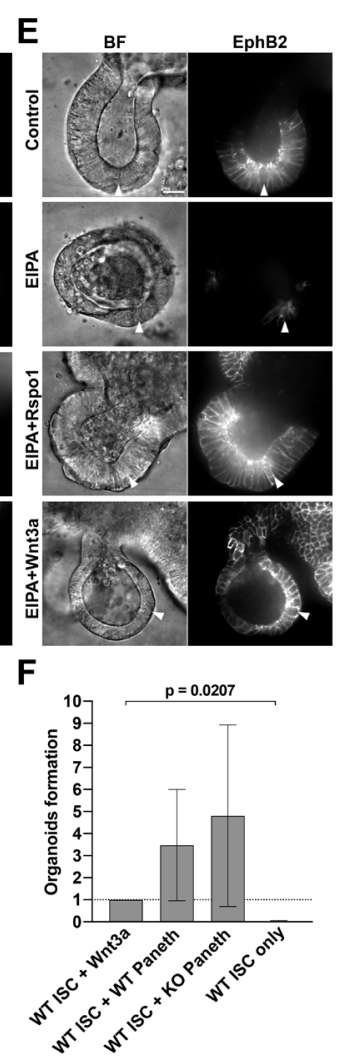
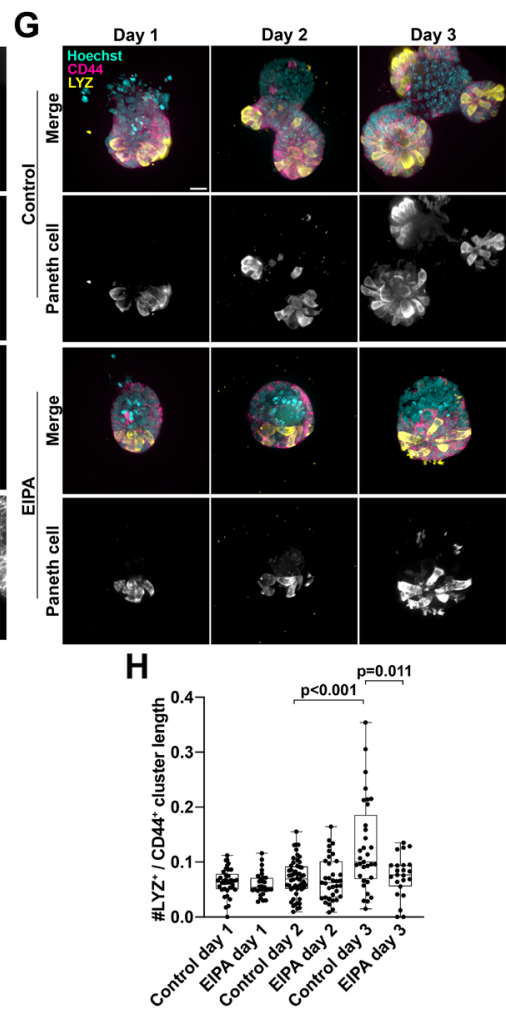

Fig. 3. Exogenous WNT rescues crypt budding impaired with loss of NHE1 activity. (A to

D) Representative confocal images and quantification of budded crypts at day 3 in $\operatorname{Lgr} 5^{\text {DTR-GFP }}$ organoids in the absence (Control) and presence of EIPA (A and B) and with Dox-inducible

NHE1 CRISPR-Cas9 silencing (C and D) shown without and with increased Rspondin1 or exogenous Wnt3a. BF, bright-field view; Lgr5, Lgr5 ${ }^{+}$ISC. Data are from 5 preparations in the absence and presence of EIPA, and 3 preparations with the NHE1 silencing. Data are statistically analyzed by the Mann-Whitney test. (E) Representative confocal images from 3 cell preparations of EphB2 immunolabeled organoids in the absence and presence of EIPA and with EIPA with increased Rspondin1 or exogenous Wnt3a. BF, brightfield; arrowheads, Paneth cells in a crypt region. Quantified EphB2 immunolabeling in fig. S3. (F) Relative efficiency of Lgr5 ${ }^{+}$ISCPaneth cell single cell reassociation, determined by the number of organoids formed. Data are normalized to the positive control (single $\mathrm{Lgr}^{+}{ }^{+}$ISCs alone with Wnt3a) and show the means of 2 independent preparations, with statistical analysis by the Wilcoxon test. (G and $\mathbf{H})$ Paneth cell 
abundance in crypts during organoid development from day 1 to day 3. (G) Representative 3D confocal images show Paneth cells in organoid crypts at the indicated days after plating without (Control) and with EIPA (n=3). 3D images show surface view of $\mathrm{z}$-stack reconstruction along the z-axis. (H) Quantification of Paneth cell numbers from conditions described (G) (n=3; MannWhitney test and two-sided student's t-test). All box plots are minimum to maximum, the box shows 25 th-75th percentiles, and the median is indicated as a central line. Data in all box plots with a statistically significant difference specified with $\mathrm{p}$ values, otherwise are not significantly different. All scale bars represent $20 \mathrm{~mm}$. 

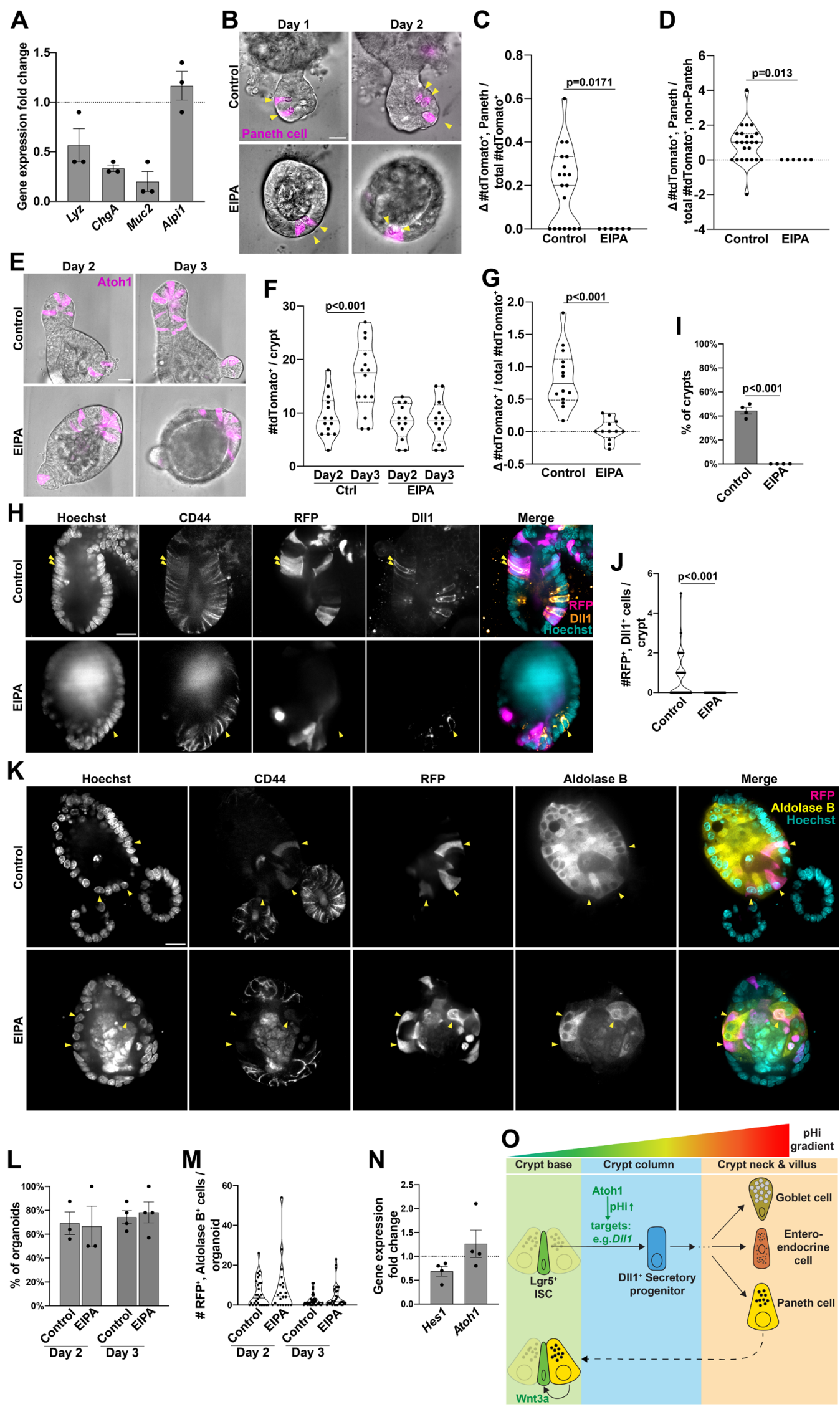
Fig. 4. Loss of NHE1 activity impairs specification of secretory but not absorptive lineage. (A) Cell marker expression (mean \pm SEM) EIPA-treated organoids relative to controls $(\mathrm{n}=3)$. Lyz, lysozyme (Paneth cell), ChgA, chromogranin A (enteroendocrine cell), Muc2, mucin 2 (goblet cell), AlpiIl, alkaline phosphatase (enterocyte). (B to D) Lineage tracing for newly produced Paneth cells from the secretory progenitors during the development of $A t o h 1^{\text {CreERT2 }} ;$ Rosa $26^{\text {tdTomato }}$ organoids in the absence (Control) and presence of EIPA. Organoids are incubated with 4-hydroxytamoxifen for $24 \mathrm{~h}$, added on day 1 followed by washing and reseeding organoids on day 2 . Reseeded organoids are then maintained for 2 days in the absence or presence of 5mM EIPA during which time-lapse imaging is used to track Paneth cell production. (B) Representative frames (day 1 and day 2) from the time-lapse recordings show Paneth cells indicated by the presence of visible dense granules (arrowhead) that are also marked by the secretory lineage marker Atoh1 (tdTomato ${ }^{+}$) to show newly produced Paneth cells from secretory progenitors. (C and D) Changes (from day 1 to day 2) of newly produced Paneth cell number in the crypt region in the absence (Control) and presence of EIPA. Data from 3 separate preparations are normalized to either the number of all secretory cells $\left(\right.$ tdTomato $\left.^{+}\right)(\mathrm{C})$, or the number of non-Paneth secretory cells (tdTomato ${ }^{+}$, non-Paneth) (D) on day 1. Statistical analysis by Mann-Whitney test. (E to G) Lineage tracing for secretory cells in $A t o h 1^{\text {CreERT2 }} ;$ Rosa $26^{\text {tdTomato }}$ organoids during development in the absence (Control) and presence of EIPA. Organoids are untreated (Control) or treated with $5 \mathrm{mM}$ EIPA on day 0 (seeding) and then incubated with 4-hydroxytamoxifen added on day 1 followed by 2-day time-lapse imaging to track Atoh $1^{+}$secretory cells. (E) Representative frames (day 2 and day 3) from a time-lapse recording of 3 preparations show secretory cells indicated by Atoh1 $\left(\right.$ tdTomato $\left.^{+}\right)$in organoids without and with EIPA. Time-lapse movie of tdTomato ${ }^{+}$secretory cell production (see movies S3, A and B). (F) Quantification of Atoh1 $\left(\right.$ tdTomato $^{+}$) labeled secretory cells from day 2 (first day for lineage tracing) to day 3 within a crypt region in the absence (Control) and presence of EIPA ( $n=3$, two-sided student's t-test). (G) Changes (from day 2 to day 3 ) of newly produced secretory cell numbers in the crypt region in the absence (Control) and presence of EIPA. Data are normalized to all secretory cells (tdTomato ${ }^{+}$) seen on day 2 ( $\mathrm{n}=3$, two-sided student's t-test). (H to $\mathbf{J})$ Lineage tracing for secretory progenitors from $\mathrm{Lgr}^{+}$ISCs using 
Lgr $5^{\mathrm{CreER}} ; \operatorname{Rosa} 26^{\mathrm{RFP}}$ organoids. (H) Representative images from 4 preparations, show double labeling of Lgr5 $^{+}$ISC progeny expressing RFP and immunolabeled for Dll1 (arrowhead) in the crypt region, indicated by $\mathrm{CD}_{4} 4^{+}$immunolabeling, of day 2 control but not EIPA-treated organoids. (I) The frequency (mean $\pm \mathrm{SEM}$ ) of $\mathrm{Lgr}^{+}$ISC labeled crypt region at day 2 organoids containing $\mathrm{RFP}^{+} / \mathrm{Dll1}^{+}$cells in the absence (Control) and presence of EIPA ( $\mathrm{n}=4$, Mann-Whitney test). (J) Quantification of the number of $\mathrm{RFP}^{+} / \mathrm{Dlll}^{+} \mathrm{g}$ cells in the crypt region in day 2 organoids maintained in the absence (Control) and presence of EIPA ( $n=4$, Mann-Whitney test). ( $\mathbf{K}$ to $\mathbf{M}$ ) Lineage tracing for enterocytes in the absorptive cell fate from $\operatorname{Lgr} 5^{+}$ISCs by using $\operatorname{Lgr} 5^{\mathrm{CreER}} ; \operatorname{Ros} a 26^{\mathrm{RFP}}$ organoids. (K) Representative images from 4 preparations show double labeling of $\mathrm{Lgr}^{+}$ISC progeny expressing RFP using and immunolabeled for Aldolase B (arrowhead) in the villus region, indicated by CD44-, of day 3 organoids without (Control) and with EIPA. (L) The frequency (mean $\pm \mathrm{SEM}$ ) at which cells in $\mathrm{Lgr}^{+}$ISC labeled villus region in day 2 and day 3 organoids are $\mathrm{RFP}^{+}$, Aldolase $\mathrm{B}^{+}$(n=4, Mann-Whitney test). (M) Quantification of the number of $\mathrm{RFP}^{+}$, Aldolase $\mathrm{B}^{+}$double-labeled cells in the villus region in day 2 and day 3 organoids maintained in the absence (Control) and presence of EIPA ( $n=4$, Mann-Whitney test). (N) Relative gene expression (mean \pm SEM) of the Notch signaling pathway in control and EIPA-treated organoids $(n=3)$. (O) Schematic representation of the main findings. All violin plots are minimum to maximum, the dashed line shows 25 th-75th percentiles, and the median is indicated as a central line. Data in all violin plots with a statistically significant difference are specified with a $\mathrm{p}$ value, otherwise are not significantly different. All scale bars, $20 \mathrm{~mm}$. 


\section{Supplementary Materials for}

\section{Intracellular pH dynamics regulates intestinal stem cell fate}

Yi Liu $^{1,2}$, Efren Reyes ${ }^{3} \uparrow$, David Castillo-Azofeifa ${ }^{3} \uparrow$, Ophir D. Klein ${ }^{3}$, Todd Nystul ${ }^{2 *} \uparrow$, Diane L. Barber ${ }^{1 * \dagger}$

Correspondence to: diane.barber@ucsf.edu; todd.nystul@ucsf.edu

This supplemental information includes:

Materials and Methods

Figs. S1 to S4

Captions for Movies S1 to S3

Other Supplementary Materials for this manuscript include the following:

Movies S1 to S3 


\section{Materials and Methods}

\section{DNA constructs and CRISPR design}

The pLX304 lenti-mCherry-SEpHluorin plasmid was generated by inserting an mCherrySEpHluorin fragment from an mCherry-SEpHluorin plasmid (Addgene \#32001) into lentiviral vector pLX304 (Addgene \#25890). The mCherry-SEpHluorin fragment was first amplified and then cloned into the Gateway entry vector $\mathrm{pENTR/D-TOPO} \mathrm{(Thermofisher,} \mathrm{catalog)} \mathrm{to} \mathrm{build}$ pENTR/D-mCherry-SEpHluorin. The mCherry-SEpHluorin fragment was then transferred from pENTR/D-mCherry-SEpHluorin into pLX304 via LR gateway reaction to build pLX304 lentimCherry-SEpHluorin. To generate the pTLCV2 NHE1 KO plasmid, guide RNA targeting the first exon of the mouse NHE1 gene was designed and cloned into an all-in-one doxycycline (Dox)-inducible vector TLCV2 (Addgene \#87360). In brief, gRNA oligo forward (5'caccgAACTTAATCATTGAACATGG-3') and reverse (5'aaacCCATGTTCAATGATTAAGTTc-3') were first annealed and then ligated into BsmBI digested TLCV2 vector.

\section{Small intestinal organoids and organoid culture}

WT, $\operatorname{Lgr} 5^{\text {DTR-GFP }}$ organoids ${ }^{47}, \operatorname{Lgr} 5^{\text {CreER}} ; \operatorname{Ros} a 26^{\text {RFP }}$ organoids $^{33}$, and Atoh $1^{\text {CreERT2 }} ;$ Rosa $26^{\text {tdTomato }}$ organoids ${ }^{33}$ were obtained from adult mice. The mCherrySEpHluorin and inducible NHE1 CRISPR-Cas9 KO organoids were generated by infecting WT organoids with lentivirus made from pLX304 lenti-mCherry-SEpHluorin and pTLCV2 NHE1 KO plasmids, respectively, followed by antibiotic selection using $10 \mathrm{mg} / \mathrm{mL}$ blasticidin for mCherry-SEpHluorin and $2 \mathrm{mg} / \mathrm{mL}$ puromycin for NHE1 CRISPR-Cas9 organoids, as described $^{48}$. Single clones of NHE1 silenced organoids were established from selected individual puromycin-resistant organoids.

The organoid cultures were generated and maintained as previously described ${ }^{8,49}$. Briefly, organoids were embedded in Matrigel (Corning, 356231) and cultured in a standard 24-well plate. For confocal imaging, organoids embedded in Matrigel were seeded into 24-well glassbottom MatTek plates (MatTek, P24G-0-10-F). Unless stated otherwise, organoids were grown in ENR medium containing advanced DMEM/F12 (Invitrogen, 12634-028) supplemented with 50 ng/mL EGF (Sigma-Aldrich, E9644-.2MG), 100 ng/mL Noggin (R\&D, 6057-NG/CF), Rspondin1 (CM, CCHMC RT0457, 1.6\% v/v), 10 mM HEPES (Invitrogen, 15630-080), $1 \mathrm{mM}$ $\mathrm{N}$-acetylcysteine (Sigma-Aldrich, A7250), 1X glutaMAX (Invitrogen, 35050-061), 1X N2 supplement (Invitrogen, 17502-048), 1X B27 supplement (Invitrogen, 17504-044), and 1X pen/strep. To induce spheroid formation, organoids were cultured in ENRWN medium, which includes ENR medium with 50\% v/v Wnt3a (CM, ATCC CRL-2647) and $10 \mathrm{mM}$ Nicotinamide (Sigma-Aldrich, N1630-100MG). For post-infection cultures, medium with an additional $10 \mathrm{mM}$ of the Rho kinase inhibitor Y-27632 (Sigma-Aldrich, Y0503-1MG) in ENRWN was used for recovery. For WNT rescue, ENR medium was supplemented with $6.4 \% \mathrm{v} / \mathrm{v}$ R-spondin 1 (CM, CCHMC RT0457) or 20\% v/v Wnt3a (CM, ATCC CRL-2647). For single-cell reassociation assays, ISC-Paneth pairs were grown in single-cell growth medium, which was ENR with 10 mM Nicotinamide, 2.5 mM Y-27632, 2.5 mM Chir99021 (Sigma-Aldrich, SML1046-5MG), 1 mM Jagged-1 (Anaspec, AS-61298), 2.5 mM Thiazovivin (Selleckchem, S1459).

$\underline{\text { NHE1 CRISPR-Cas9 silencing and validation }}$ 
To induce and validate NHE1 CRISPR-Cas9 editing, $2 \mathrm{mg} / \mathrm{mL}$ doxycycline (Dox) was added to the ENR medium and to Matrigel after passaging organoids. After induction for 2-3 days, clones containing at least $80 \%$ Cas9-EGFP positive organoids were selected for genomic DNA (gDNA) extraction. The gDNA was isolated from selected clonal organoids using the gDNA tissue miniprep system (Promega, A2051) and evaluated by PCR and DNA gel electrophoresis using primer pair forward (5'- GCCCGTGGTCCAGCCTATC-3') and reverse (5'-GTCCCATCCCAGCTGTAGGAGA-3'). Purified PCR fragment containing the edited DNA was sequenced by Sanger sequencing using primer forward (5'CGTCTGGGGATTTCATCCACCT-3') and reverse (5'CTATCTTCATGAGGCAGGCCAGGA-3') respectively. The sequencing results were analyzed by comparing WT and -Dox samples (Extended Data Figure 2).

\section{pHi determination}

Ratiometric imaging of the pHi biosensor mCherry-SEpHluorin was performed as described $^{14}$. In brief, organoids embedded in Matrigel were cultured in ENR medium supplemented with DMSO vehicle $(1: 1,000)$ or $5 \mathrm{mM}$ EIPA for 1 to 3 days. Before imaging, growth medium was replaced with freshly made pHi buffer containing $25 \mathrm{mM} \mathrm{NaHCO}, 115$ $\mathrm{mM} \mathrm{NaCl}, 5 \mathrm{mM} \mathrm{KCl}, 10 \mathrm{mM}$ glucose, $1 \mathrm{mM} \mathrm{K}_{3} \mathrm{PO}_{4}, 1 \mathrm{mM} \mathrm{MgSO}_{4}$, and $2 \mathrm{mM} \mathrm{CaCl}_{2} \mathrm{pH} 7.4$ and including the membrane dye CellMask ${ }^{\mathrm{TM}}$ (Molecular probes, C10046) for $10 \mathrm{~min}$. After washing with fresh $\mathrm{pHi}$ buffer not containing CellMask ${ }^{\mathrm{TM}}$ fluorescence images were acquired using a customized spinning disk confocal (Yokogawa CSU-X1) on a Nikon Ti-E microscope equipped with a live-cell imaging chamber maintained with $5 \% \mathrm{CO}_{2}$ at $37^{\circ} \mathrm{C}$, a $40 \mathrm{X}$ water objective, $488 \mathrm{~nm}, 560 \mathrm{~nm}$, and $590 \mathrm{~nm}$ excitation lasers. and a Photometrics cMYO cooled CCD camera. Images were stored as Z-stacks of $2 \mathrm{~mm}$-optical sections. To calibrate fluorescence ratios of mCherry-SEpHluorin to $\mathrm{pHi}$, after each experiment, organoids were incubated for 15-20 min with a $\mathrm{KCl}$ buffer $\left(80 \mathrm{mM} \mathrm{KCl}, 50 \mathrm{mM} \mathrm{K}_{3} \mathrm{PO}_{4}, 1 \mathrm{mM} \mathrm{MgCl}_{2}\right)$ containing $20 \mathrm{mM}$ nigericin (Invitrogen, N1495) at $\mathrm{pH}$ 7.8. After acquiring fluorescence ratios, organoids were washed and incubated with nigericin buffer at $\mathrm{pH} 6.6$ and fluorescence ratios were again acquired to generate a 2-point calibration conversion.

For image analysis, backgrounds were removed from $\mathrm{Z}$ stacks and single optical sections containing representative intestinal crypts were chosen for quantification. Individual cells were segmented by the membrane dye. To identify major cell types in the crypt, Paneth cells were selected based on size and presence of visible intracellular granules under both bright-field and mCherry channels, ISCs were identified based on their location which is intermingled between two Paneth cells, other cells along the crypt column starting at a position two-cell above the upmost Paneth cell and ending at the crypt neck, the upmost part of a budded crypt, were further specified as the crypt column and the crypt neck. In the crypt without a bud, up to four of the cells closest to the upmost Paneth cell were chosen as the column for $\mathrm{pHi}$ measurement. Because the crypt region and villus region were less distinguishable by live-cell imaging in the day 1 organoid during development, the column region was not specified for $\mathrm{pHi}$ determination on day 1. To calculate $\mathrm{mCherry/SEpHluorin}$ intensity ratios, fluorescence intensity was measured in the selected individual Paneth cells, ISCs, and cells located in the column and neck using ImageJ. In Microsoft Excel, the pHi values were determined by applying those intensity ratios to a standard curve generated by graphing the nigericin $\mathrm{pH}$ values ( $\mathrm{Y}$-axis) against their dependent intensity ratios (X-axis). Because of the $\mathrm{mCherry} / \mathrm{SEpHluorin}$ intensity ratio in the cells in the organoid 
villus (above the crypt neck) was too high to be calibrated, the pHi in those cells were not determined.

\section{Crypt budding quantification and imaging}

Under a bright-field view on the spinning disc confocal microscope, a budded crypt was defined by the epithelial protrusion (containing visible Paneth cells) grown out of the spherical cyst with the extension protruded larger than the thickness of the epithelial layer of the cyst. To quantify crypt budding, the number of budded crypts were counted in organoids under each of the following conditions. (1) For control vs EIPA conditions, day 3 organoids grown in ENR medium supplemented with DMSO (vehicle control) or $5 \mathrm{mM}$ EIPA were scored for crypt budding. (2) For inducible CRISPR-Cas9 mediated NHE1 silencing, organoids in ENR medium were treated with or without Dox for 2 days and then re-seeded in ENR with or without Dox for another 3 days before quantification. (3) For WNT rescue experiments, EIPA-treated and CRISPR-Cas9 silenced organoids were cultured in the ENR medium supplemented with either $3 \mathrm{X}$ Rspol or $20 \%$ exogenous Wnt3a for 3 days prior to quantification. To imaging crypt budding in the $\operatorname{Lgr} 5^{\text {DTR-GFP }}$ organoids (Figure 2A), a 3-day time-lapse imaging (bright-field, FITC; 40X) began immediately after seeding (day 0) in ENR + DMSO (vehicle control) and ENR $+5 \mathrm{mM}$ EIPA. Images were acquired as Z-stacks of $2 \mathrm{~mm}$ optical sections every $3 \mathrm{hrs}$. To imaging pHi dynamics in crypt budding in the mCherry-SEpHluorin organoids, a 2-day time-lapse imaging (bright-field, FITC, PE-Texas red; 20X) was taken one day after seeding and each 2uM Z-stack image set was acquired every 30 mins.

\section{Proliferation assay and crypt cell quantification}

Click-iT Edu cell proliferation kit (Invitrogen, C10340) was used to assess crypt proliferation. Day 3 organoid culture was first incubated in $10 \mathrm{mM}$ Edu solution for 2 hours then proceeded for immunostaining. To quantify the proliferative cells within CD $44^{+}$clusters, numbers of $\mathrm{Edu}^{+}$and $\mathrm{CD} 44^{+}$positive cells in each CD44 labeled cluster were first counted separately from confocal z-plane sectioning through the middle of a budded crypt or an unbudded organoid. For normalization, a ratio of numbers of $\mathrm{Edu}^{+}$; $\mathrm{CD}_{4} 4^{+}$double-positive cells to the numbers of total $\mathrm{CD} 44^{+}$cells were then calculated as a measurement of overall proliferation. To quantify other crypt cells, the numbers of $\mathrm{Lgr}^{+}$ISCs and $\mathrm{Lgr5} 5^{-}$CD $44^{+}$cells (crypt progenitors) within each $\mathrm{CD} 44^{+}$cluster were counted and normalized to the total number of CD44+ cells, in the same manner as the proliferation assay. To count Paneth cells in developing organoids, organoids were fixed at day1, day2, and day3 for immunostaining. All $\mathrm{LYZ}^{+}$Paneth cells present in each $\mathrm{CD} 44^{+}$cluster were counted in $3 \mathrm{D}$ confocal images and normalized to the arc length of the 3D projection of $\mathrm{CD} 44^{+}$clusters.

\section{Immunolabeling}

Matrigel embedded organoids were fixed in 2\% paraformaldehyde for $45 \mathrm{~min}$ at RT. Fixed organoids were washed $3 \times 20$ min at RT with 1 X Glycine-PBS $(7.5 \% \mathrm{~m} / \mathrm{v})$ followed by washing 2x10 min with PBS. Organoids were then permeabilized in $0.5 \%$ Triton X-100 for 15 min at RT and blocked in PBS buffer containing $0.1 \%$ BSA, $0.2 \%(\mathrm{v} / \mathrm{v})$ Triton X-100, and $0.04 \%$ (v/v) Tween-20, 5 10\% (v/v) serum overnight at $4{ }^{\circ} \mathrm{C}$. Organoids were incubated overnight at $4{ }^{\circ} \mathrm{C}$ with primary antibodies diluted in blocking buffer. Antibodies and dilutions included anti-CD44 (BioLegend, 103030, 1:500), anti-lysozyme (LYZ) (Dako, EC 3.2.1.17,1:100), anti-EphB2 (R\&D, AF496,1:25), anti-Dll1(R\&D, AF5026. 1:14), and anti-Aldolase B (Abcam, ab75751, 
1:200). After washing with blocking buffer (w/o serum) 3x1 hr at RT, organoids were incubated overnight at $4^{\circ} \mathrm{C}$ with fluorescent secondary antibodies (Molecular Probes) diluted 1:200 in blocking buffer. After incubation, organoids were washed 3x1 hr with blocking buffer (w/o serum) and then 20 min with PBS. Washed organoids were stained with Hoechst 33342 (Molecular probes, 1:1000) and stored in PBS at $4^{\circ} \mathrm{C}$. Fluorescence images were acquired using a customized spinning disk confocal (Yokogawa CSU-X1) on a Nikon Ti-E microscope with a $40 \mathrm{X}$ water objective equipped with a Photometrics cMYO cooled CCD camera.

For image analysis, backgrounds were removed from $\mathrm{Z}$ stacks and single optical sections containing representative intestinal crypts were chosen for quantification. Individual cells were segmented by the membrane dye. To identify major cell types in the crypt, Paneth cells were selected based on size and presence of visible intracellular granules under both bright-field and mCherry channels, ISCs were identified based on their location which is intermingled between two Paneth cells, other cells along the crypt column starting at a position two-cell above the upmost Paneth cell and ending at the crypt neck, the upmost part of a budded crypt, were further specified as the crypt column and the crypt neck. In the crypt without a bud, up to four of the cells closest to the upmost Paneth cell were chosen as the column for $\mathrm{pHi}$ measurement. Because the crypt region and villus region were less distinguishable by live-cell imaging in the day 1 organoid during development, the column region was not specified for $\mathrm{pHi}$ determination on day 1. To calculate mCherry/SEpHluorin intensity ratios, fluorescence intensity was measured in the selected individual Paneth cells, ISCs, and cells located in the column and neck using ImageJ. In Microsoft Excel, the pHi values were determined by applying those intensity ratios to a standard curve generated by graphing the nigericin $\mathrm{pH}$ values ( $\mathrm{Y}$-axis) against their dependent intensity ratios (X-axis). Because of the mCherry/SEpHluorin intensity ratio in the cells in the organoid villus (above the crypt neck) was too high to be calibrated, the pHi in those cells were not determined.

Flow cytometry and single cell reassociation assay

NHE1 silencing was induced by adding $2 \mathrm{mg} / \mathrm{mL}$ Dox to the inducible-NHE1 CRISPR Cas9-GFP organoids culture for 2 days. On the day of sorting, the NHE1 CRISPR silenced organoids and $\operatorname{Lgr} 5^{\text {DTR-GFP }}$ organoids were first dissociated and then stained for CD44 (BioLegend, 103030, 1:250), CD24 (BD, 553262, 1:250), and Live/Dead (Invitrogen, L23105, 1:1000). Fluorescence-activated cell sorting (FACS) was performed on the BD FACSAira. Live $\mathrm{Lgr}^{+}$ISCs (Lgr5-DTR-GFP ${ }^{\text {high }}$, CD $44^{\text {high }}$, CD $24^{\text {low }}$, and side-scatter ${ }^{\text {low }}$ ) were sorted and mixed with either the sorted live WT Paneth cells (Lgr5-DTR-GFP negative CD44 ${ }^{\text {high }}$, CD24 $4^{\text {high }}$, and sidescatter $^{\text {high }}$ ) or the sorted live NHE1-silenced Paneth cells (Cas9-GFPhigh, CD44 ${ }^{\text {high }}$, CD24 ${ }^{\text {high }}$, and side-scatter $\left.{ }^{\text {high }}\right)$ at 1:1 ratio (400-500 cells each) as previously described ${ }^{30}$. In addition, groups containing the $\mathrm{Lgr}^{+}$ISCs alone, the $\mathrm{Lgr}^{+}$ISCs + Wnt3a $(20 \% \mathrm{v} / \mathrm{v}$ Wnt3a in single-cell growth media), the WT Paneth cells alone, and the NHE1-silenced Paneth cells alone were also included as controls for normalization. All groups were grown in single cell growth media for 3 days and then switched to normal ENR media. To evaluate organoid formation ability from the ISCPaneth cell pairs, the number of organoids formed in all the groups (if any) was counted on day 8 and normalized to the $\mathrm{Lgr}^{+}$ISCs + Wnt3a group.

\section{Lineage tracing}


In general, lineage tracing in the Atoh $1^{\text {CreERT2}} ;$ Rosa $26^{\text {tdTomato }}$ and in the $\operatorname{Lgr} 5^{\text {CreER}}$; Rosa $26^{\mathrm{RFP}}$ organoids were prepared by treating the day 1 organoids with $0.1 \mathrm{mM} 4$ hydroxytamoxifen (Sigma-Aldrich, 579002) for 26 hours followed by washing and passaging. Reseeded organoids were cultured with vehicle control or 5mM EIPA for 2-3 days either in a standard culture incubator before immunostaining for D111 and Aldolase B, or in a live-cell imaging chamber for confocal microscopy for Paneth cell production, recorded every $24 \mathrm{hr}$, with z-stacks of $2 \mathrm{~mm}$ optical sections covering the entire crypt region. To reveal overall Atoh $1^{+}$ secretory cell production, day 1 control and EIPA-treated Atoh $1^{\text {CreERT2 }}$; Rosa $26^{\text {tdTomato }}$ organoids culture were added with $0.1 \mathrm{mM}$ TAM for 24 hours first before starting a $48 \mathrm{hr}$ time-lapse imaging (day 2 to day 3), which were acquired at 30-min intervals, in a 2-mm z step size per optical section. To quantify lineage tracing, numbers of $\mathrm{RFP}^{+}$or tdTomato ${ }^{+}$cells were counted in the crypt regions, indicated by immunolabelled CD $44^{+}$clusters in the $\operatorname{Lgr} 5^{\mathrm{CreER}} ;$ Rosa $26^{\mathrm{RFP}}$ organoids, or by clusters of live Atoh $1^{+}$cells and Paneth cells in the Atoh $1^{\text {CreERT2 }}$; Rosa $26^{\text {tdTomato }}$ organoids. To assess the Paneth cell production, changes of newly generated Atoh $1^{+}$Paneth cells $\left(\mathrm{RFP}^{+} /\right.$dense granule $\left.{ }^{+}\right)$numbers within $24 \mathrm{hrs}$ were normalized to the number of total Atoh $1^{+}$ cells $\left(\mathrm{RFP}^{+}\right)$as well as the Atoh $1^{+}$non-Paneth cells $\left(\mathrm{RFP}^{+} /\right.$dense granule $\left.{ }^{-}\right)$. Similarly, for measuring the overall secretory cell production, changes of newly produced Atoh $1^{+}$cells $\left(\mathrm{RFP}^{+}\right)$ numbers from day2-3 were normalized to the number of Atoh $1^{+}$cells seen on day 2 . To determine differentiation capability of $\mathrm{Lgr}^{+}$ISCs into $\mathrm{Dll1}^{+}$enriched secretory progenitors, the Paneth cells, and the enterocytes, frequencies of crypts containing $\mathrm{RFP}^{+} / \mathrm{CD} 44^{+} / \mathrm{Dlll}^{+}$(secretory progenitors), $\mathrm{RFP}^{+} / \mathrm{CD}_{4} 4^{+} / \mathrm{LYZ}^{+}$(Paneth cells), and organoids containing $\mathrm{RFP}^{+} / \mathrm{CD}^{-} / 4^{-} /$Aldolase $\mathrm{B}^{+}$cells were calculated. The number of $\mathrm{RFP}^{+} / \mathrm{CD}_{4} 4^{+} / \mathrm{D}_{11} 1^{+}, \mathrm{RFP}^{+} / \mathrm{CD} 44^{+} / \mathrm{LYZ}^{+}$, and $\mathrm{RFP}^{+} / \mathrm{CD} 44^{-} /$Aldolase $\mathrm{B}^{+}$cells were also quantified in each crypt or organoid as a measurement for $\mathrm{Lgr}^{+}$ISC differentiation.

RNA extraction and quantitative reverse transcription PCR (RT-qPCR)

Total RNA was extracted from organoids using the RNeasy plus mini kit (Qiagen, 74134). RNA was used to generate cDNA using the iScript cDNA synthesis kit (Bio-Rad, 1708890). The mRNA level of target genes was accessed by RT-qPCR on a Real-time PCR system (Applied Biosystems) using the SYBR green supermix reaction (Bio-Rad, 170-8882). The relative expression of targets was analyzed using the double delta $\mathrm{C}_{\mathrm{t}}$ method by normalizing to GAPDH. Primer sequences were obtained from the references ${ }^{50-52}: \operatorname{Lgr} 5$ forward (5'GGGAGCGTTCACGGGCCTTC-3'), Lgr5 reverse (5'-GGTTGGCATCTAGGCGCAGGG-3'); $L y z$ forward (5'-GGAATGGATGGCTACCGTGG-3'), Lyz reverse (5'-

CATGCCACCCATGCTCGAAT-3'); Alpil forward (5'-AGGATCCATCTGTCCTTTGG-3'), Alpil reverse (5'-ACGTTGTATGTCTTGGACAG-3'); ChgA forward (5'CTCGTCCACTCTTTCCGCAC-3'), ChgA reverse (5'-CTGGGTTTGGACAGCGAGTC-3'); $M u c 2$ forward (5'-ATGCCCACCTCCTCAAAGAC-3'), Muc2 reverse (5'GTAGTTTCCGTTGGAACAGTGAA-3'); Hes 1 forward (5'GCTCACTTCGGACTCCATGTG-3'), Hes 1 reverse (5'-GCTAGGGACTTTACGGGTAGCA3'); Atohl forward (5'-GCCTTGCCGGACTCGCTTCTC-3'), Atoh1 reverse (5'TCTGTGCCATCATCGCTGTTAGGG-3'); Gapdh forward (5'TGTGTCCGTCGTGGATCTGA-3'), Gapdh reverse (5'-CCTGCTTCACCACCTTCTTGA-3').

$\underline{\text { Statistics }}$ 
Statistical analysis was performed using GraphPad Prism 8. Outliers were determined using the ROUT method by fitting data with nonlinear regression and were excluded from other statistical tests. Data fitting a normal distribution were analyzed using a standard t-test/Welch's ttest. The non-normally distributed data were analyzed by the Mann-Whitney ranking test.

\section{Supplementary figures}
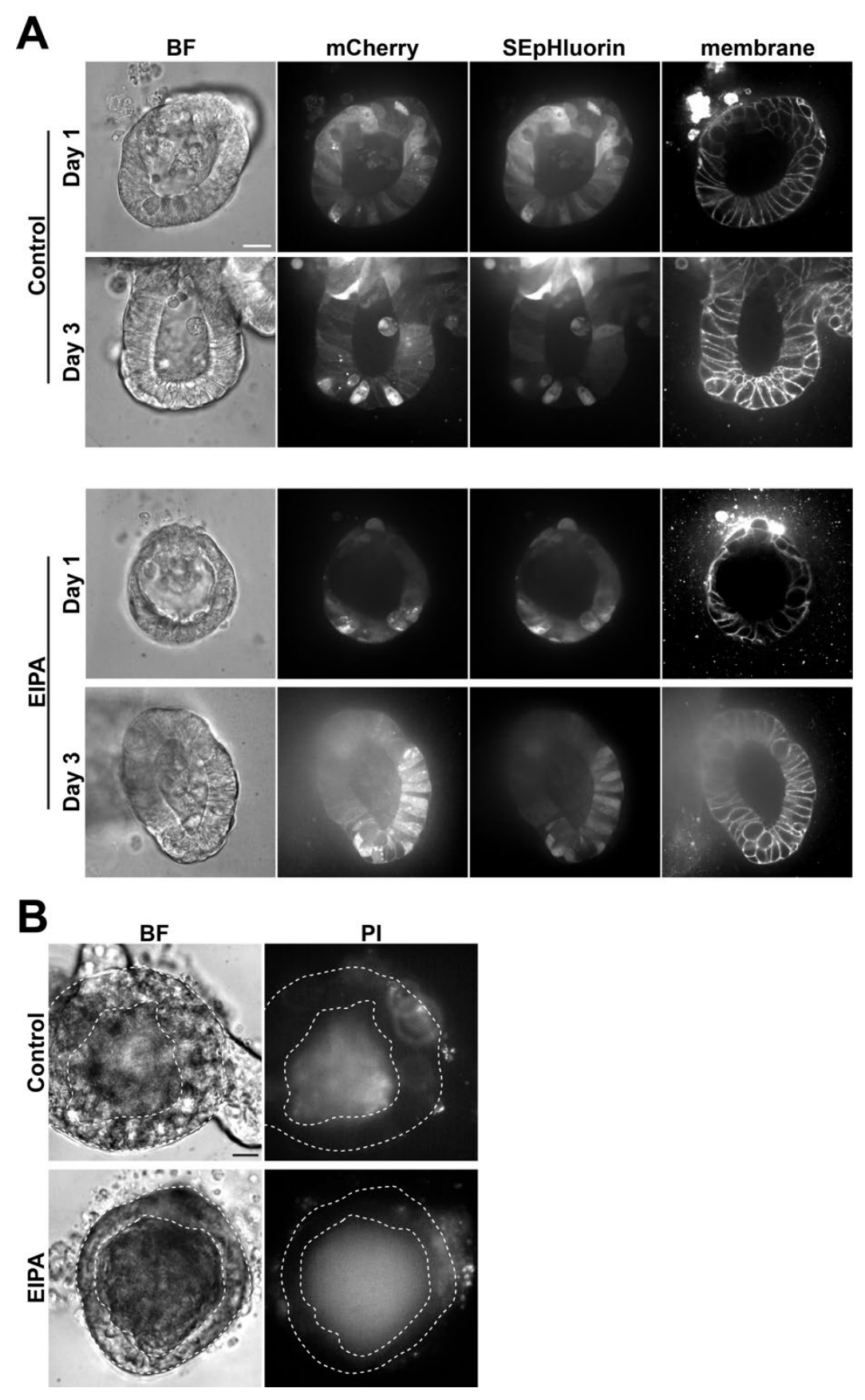
Fig. S1. mCherry-SEpHluroin and cell viability in organoids.

(A) Representative live-cell images of mCherry-SEpHluorin in the crypt region in day 1 and day 3 organoids in the absence (Control) and presence of $5 \mathrm{mM}$ EIPA. Panels show brightfield (BF) individual and merged mCherry and SEpHluorin signal, and far-red membrane dye to determine individual cells. (B) Propidium iodide (PI) staining as an index of cell viability in day 3 organoids in the absence (Control) and presence of EIPA. Space between dashed circles indicates epithelial layer. Inner dashed circle indicates the lumen containing apoptotic cells with positive staining. All scale bars, $20 \mathrm{~mm}$. 

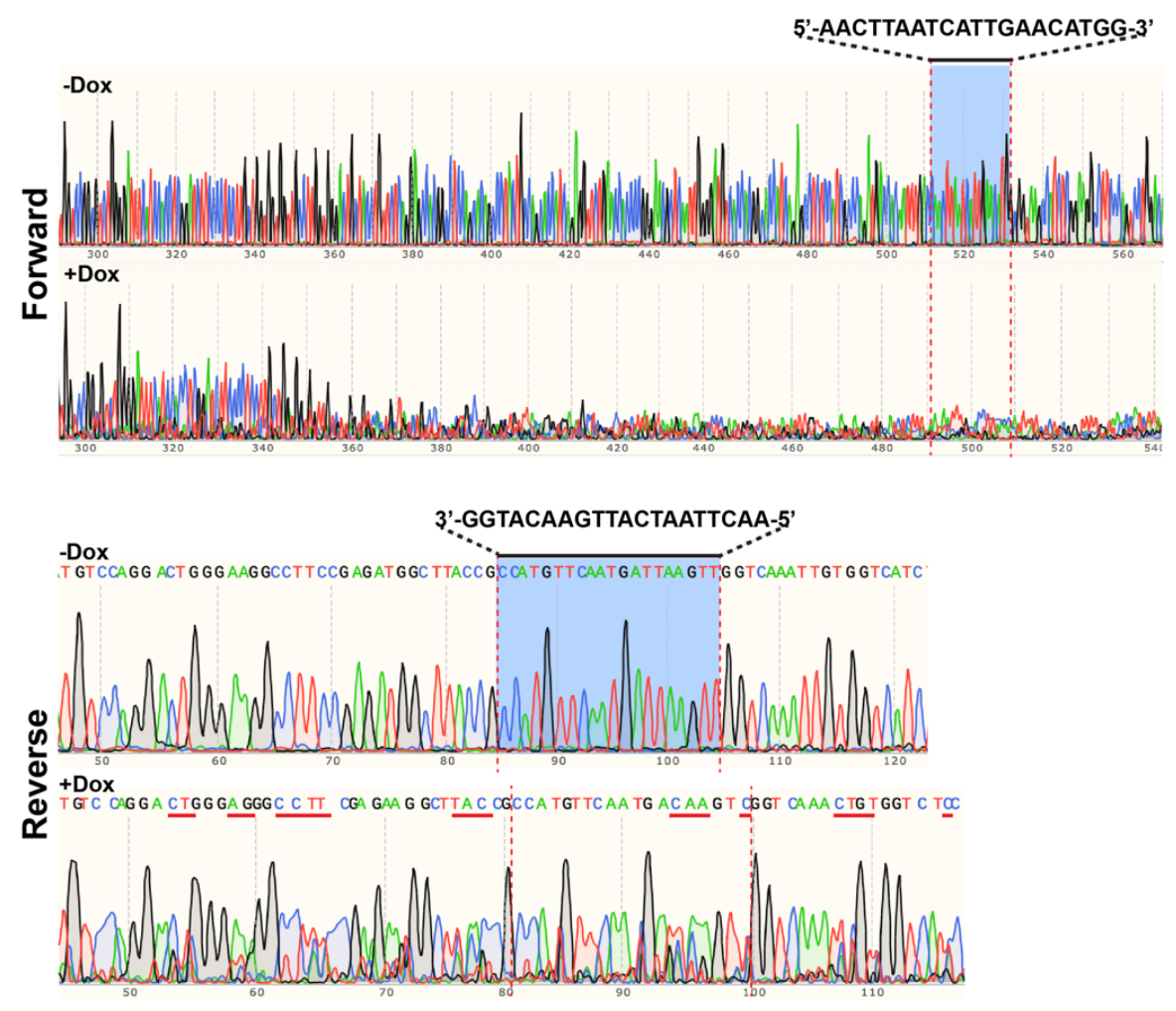

Fig. S2. Validation of induced CRISPR-Cas9 NHE1 silencing.

Comparison of Sanger sequencing of PCR fragments of NHE1 gene from non-induced (-Dox) and induced (+Dox) organoids. Results are generated using forward and reverse sequencing primers, respectively. The Cas9 guide RNA targeting region is indicated by a black line with red dashed lines. Reduction of signal in forwarding sequencing indicates large disruption of DNA sequence. A red solid line in reverse sequencing indicates CRISPR indels. 

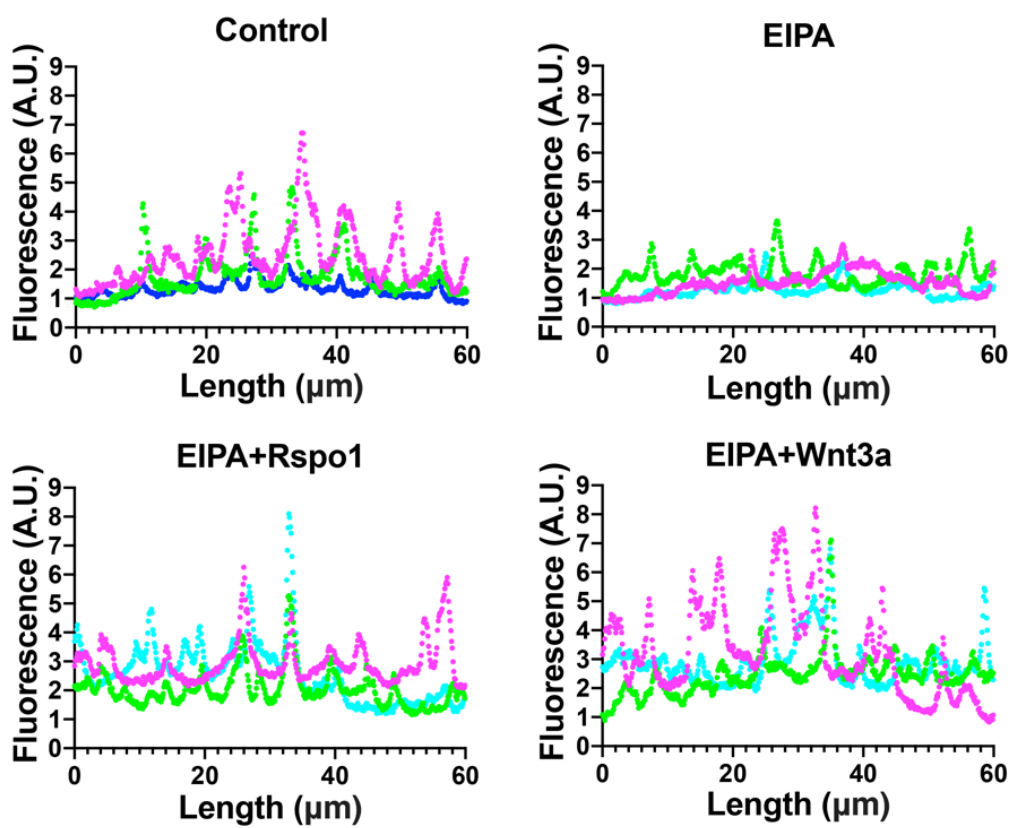

Fig. S3. Quantification of EphB2 staining in organoids.

Representative line plots of intensity of EphB2 immunolabeling in crypt region of day 3 organoids with and without WNT rescue. Fluorescence intensity is determined by drawing a line across lateral layers of crypt cells using the line tool function in ImageJ. Each plot shows 3 individual crypt regions indicated by different colors $(n=3)$. 


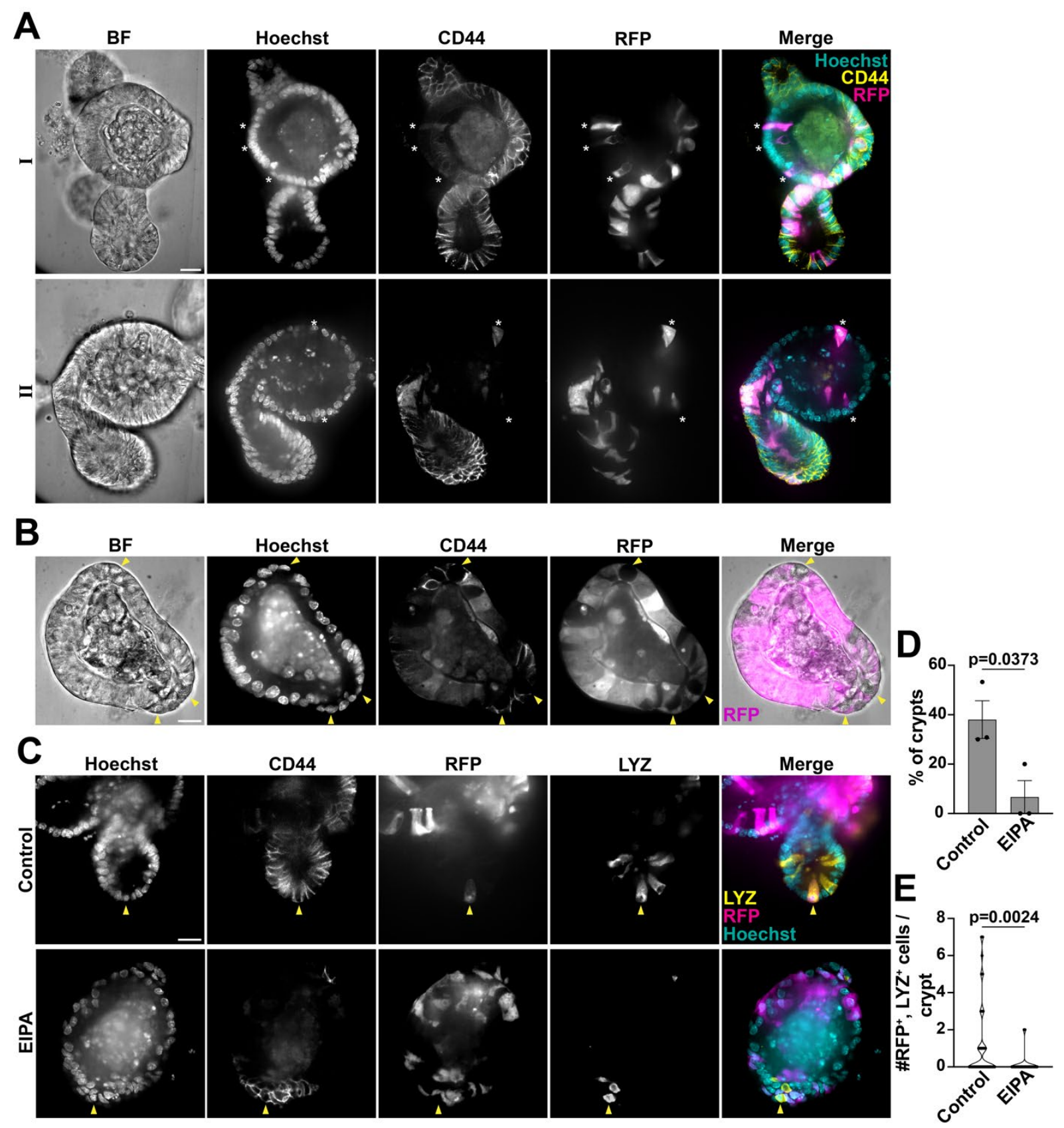

Fig. S4. Lineage tracing of crypt and villus cells in $\operatorname{Lgr} 5^{\mathrm{CreER}}$; Rosa $26^{\mathrm{RFP}}$ organoids.

(A) Representative images show the presence of labeled $\mathrm{Lgr} 5^{+}$ISC progeny $\left(\mathrm{RFP}^{+}\right)$in crypt $\left(\mathrm{CD} 44^{+}\right)$and villus $\left(\mathrm{CD} 44^{-}\right)$regions of day 3 organoids. Organoids are treated with 4hydroxytamoxifen on day 1 followed by washing and reseeding on day 2 . Immunolabeled images show 2 examples (I, II) of day 3 reseeded organoids. (B) Example of an EIPA-treated organoid with robust labeling in the $\mathrm{Lgr}^{+}$ISC progeny. Arrowhead indicates Paneth cells, which are identified by visible dense granules and large cell size. ( $\mathbf{C}$ to $\mathbf{E}$ ) Lineage tracing for Paneth cells from $\mathrm{Lgr}^{+}$ISCs before EIPA treatment. (C) Representative images show the presence of double-labeled Lgr5 ${ }^{+}$ISC progeny expressing RFP and immunolabeled with Lysozyme (LYZ) antibodies (Paneth cell) (arrowhead) in the crypt region (CD44 ${ }^{+}$) of day 3 
control but not the EIPA-treated organoids (n=3). (D) Frequency (mean \pm SEM) at which $\operatorname{Lgr}^{+}$ ISC labeled crypt region in day 3 organoids contains $\mathrm{RFP}^{+} / \mathrm{LYZ}^{+}$cells in controls and with EIPA ( $n=3$, Mann-Whitney test). (E) Quantification of the number of $\mathrm{RFP}^{+} / \mathrm{LYZ}^{+}$cells in the crypt region of day 3 organoids in the absence (control) and presence of EIPA ( $n=3$, Mann-Whitney test). The violin plot is minimum to maximum, the dashed line shows 25 th- 75 th percentiles, and the median is indicated as a central line. Data with a statistically significant difference are specified with p values. All scale bars, $20 \mathrm{~mm}$.

\section{Supplementary movie captions}

\section{Movie S1.}

Video of mCherry-SEpHluroin fluorescence ratio in a budding crypt (day 1 to day 2), corresponding to Fig. 1E. Fluorescence ratios are calibrated to $\mathrm{pH}$ values using nigericincontaining buffers to show that a dynamic pHi gradient, lower at the crypt base and higher at the crypt neck, is generated during crypt budding. Left view, bright field. Right view, ratio (mCherry/SEpHluorin).

\section{Movie S2A.}

Video of crypt budding in an untreated control $\operatorname{Lgr} 5^{\text {DTR-GFP }}$ organoid from day 0 to day 3, corresponding to Fig. 2A. The crypt region elongates and forms a budded crypt. Left view, bright field. Right view, Lgr5.

\section{Movie S2B.}

Video of impaired crypt budding in an EIPA-treated $\operatorname{Lgr} 5^{\text {DTR-GFP }}$ organoid from day 0 to day 3, corresponding to Fig. 2A. The crypt region initiates a protrusion but fails to form a budded crypt. Left view, bright field. Right view, Lgr5 ${ }^{+}$ISCs.

\section{Movie S3A.}

Video shows the production of secretory cells in the crypt region of an untreated control Atoh $1^{\text {CreERT2}} ;$ Rosa $26^{\text {tdTomato }}$ organoid from day 2 to day 3, corresponding to Fig 4E. Newly produced Atoh $1^{+}\left(\right.$tdTomato $\left.^{+}\right)$secretory cells appear in the crypt as the crypt elongates and forms a bud.

\section{Movie S3B.}

Video shows attenuated production of secretory cells in the crypt region of an EIPA-treated Atoh $1^{\text {CreERT2 }} ;$ Rosa $26^{\text {tdTomato }}$ organoid from day 2 to day 3, corresponding to Fig 4E. No evident increase of newly produced Atoh $1^{+}\left(\right.$tdTomato $\left.^{+}\right)$secretory cells appears in the unbudded crypt region. 